\title{
Transient Global Amnesia Following Coil Embolization for a Basilar-tip Aneurysm: Case Reports of Two Patients
}

\author{
Muneaki Hashimoto, ${ }^{1}$ Yoshinobu Kamio, ${ }^{1}$ Hisaya HiRAmatsu, ${ }^{1}$ \\ Shusuke MATSUI, ${ }^{1}$ Mika KAMIYA, ${ }^{2}$ and Hiroki NAMBA ${ }^{1}$ \\ ${ }^{1}$ Department of Neurosurgery, Hamamatsu University School of Medicine, Hamamatsu, \\ Shizuoka, Japan \\ ${ }^{2}$ Department of Radiology, Yaizu City Hospital, Yaizu, Shizuoka, Japan
}

\begin{abstract}
Transient global amnesia (TGA) can be caused by medications, ischemia, metabolic abnormalities, and seizures. We describe two cases of TGA following coil embolization for a basilar-tip aneurysm. A 73-year-old woman developed transient acute anterograde amnesia after coil embolization for a basilar-tip aneurysm. Diffusion-weighted imaging (DWI) revealed an ischemic lesion in the anterior nucleus of the thalamus. A 67-year-old woman developed transient acute amnesia after a stent-assisted coil embolization of a basilar-tip aneurysm. A DWI showed ischemic lesions in the anterior nucleus of the thalamus. Any ischemic changes to areas of the anterior nucleus that are fed by the thalamoperforating and premammillary arteries should be considered in a differential diagnosis for TGA in patients who have undergone coil embolization for a posterior circulation cerebral aneurysm.
\end{abstract}

Keywords: transient global amnesia, thalamic infarction, thalamoperforating artery

\section{Introduction}

Transient global amnesia (TGA) is characterized by the sudden onset of anterograde and retrograde amnesia that often lasts for approximately 6 hours, but may extend for up to 24 hours. ${ }^{1-3)}$ The differential diagnosis for TGA includes seizures, adverse reaction to certain medications, and metabolic disorders. Few studies have reported TGA after coil embolization for a posterior circulation cerebral aneurysm. Previous studies have demonstrated that the anterior nucleus of the thalamus, which is fed by the thalamoperforating artery (TPA) and the premammillary arteries, is responsible for memory function. We reported two cases of patients with transient acute amnesia following aneurysm coil embolization.

Received April 3, 2020; Accepted June 15, 2020

Copyright $\subseteq 2021$ by The Japan Neurosurgical Society This work is licensed under a Creative Commons AttributionNonCommercial-NoDerivatives International License.
Case 1

A 73-year-old woman with a history of acute myocardial infarction and arteriosclerosis obliterans underwent head magnetic resonance imaging (MRI) for reoccurring headaches. MR angiography (MRA) revealed the presence of an unruptured basilar-tip aneurysm measuring $9 \mathrm{~mm}$. She received aspirin $100 \mathrm{mg} /$ day and clopidogrel $75 \mathrm{mg} /$ day for 2 weeks prior to coil embolization. She underwent coil embolization of the aneurysm with the double catheter technique, but without balloon or stent assistance (Figs. 1A and 1B). She received 6000 units of heparin intravenously during the procedure, to maintain the activated clotting time between 250 and 300 seconds. She also received $60 \mathrm{mg}$ propofol and $60 \mathrm{mg}$ rocuronium during the procedure. A cerebral angiography performed after coil embolization depicted a single dominant TPA, known as the artery of Percheron (AOP), arising from the left posterior cerebral artery which was patent (Fig. 1C). Immediately after the procedure, she had memory loss. The patient knew her name but could not recall the date, the location, or the reason for 

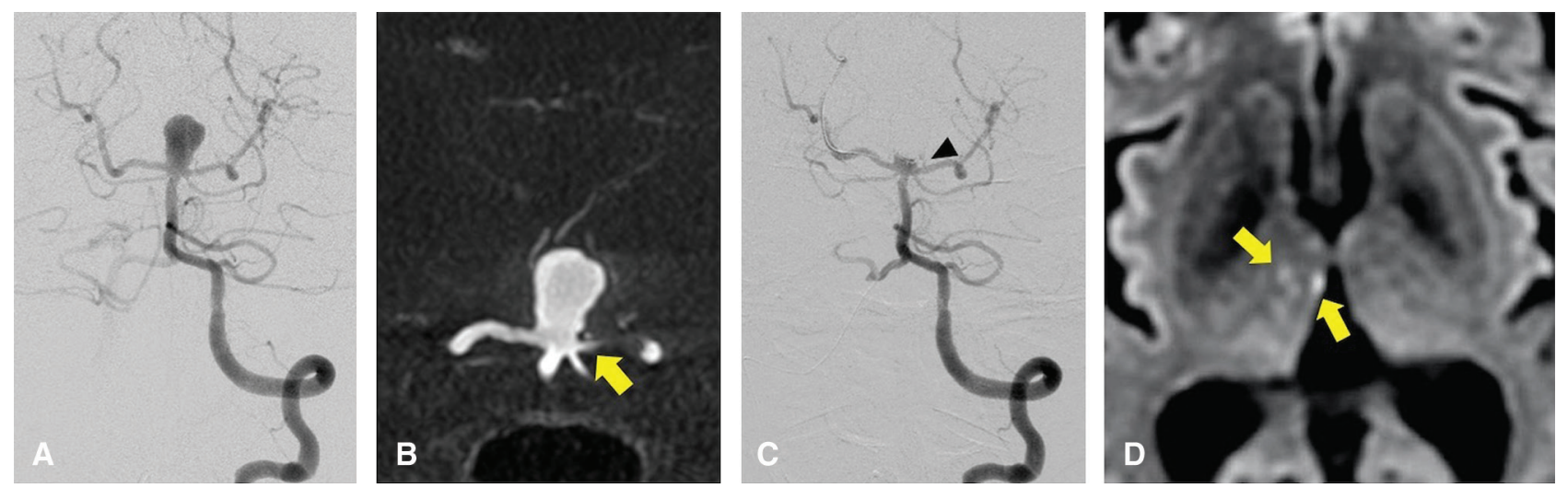

Fig. 1 (A and B) Preoperative cerebral angiography and MIP images showing basilar-tip aneurysm and a TPA from left posterior cerebral artery (arrow). (C) Postoperative cerebral angiography showing patent TPA (arrow head). (D) DWI showing high intensity within the right mesial thalamus (arrows). DWI: diffusion-weighted image, MIP: most intensive picture, TPA: thalamoperforating artery.
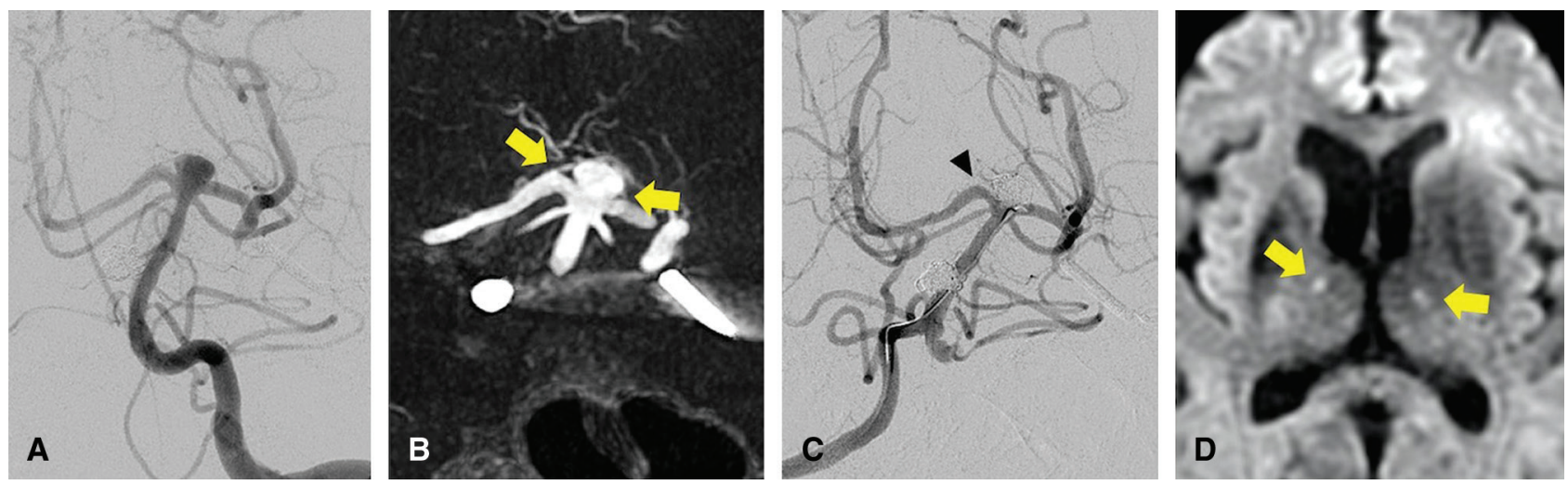

Fig. 2 (A and B) Preoperative cerebral angiography and MIP images showing basilar-tip aneurysm and thalamoperforating arteries (arrows) from bilateral PCAs. (C) Postoperative cerebral angiography showing patent TPA (arrow head). (D) DWI showing high intensity within bilateral anterior nucleus of thalamus (arrows). DWI: diffusion-weighted image, MIP: most intensive picture, PCAs: posterior cerebral arteries, TPA: thalamoperforating artery.

admission. She repeatedly asked, "Where am I and why am I here?" and could not remember her neurosurgeons whom she had met after hospitalization. She had memory loss for 4 days between the date of admission and the date of surgery about retrograde amnesia. Cranial nerves, motor strength, sensation, and cerebellar testing were normal. Upon presentation of the symptoms, she received argatroban and edaravone for ischemia. Her anterograde amnesia resolved 12 hours after coil embolization and a diffusion-weighted image (DWI) revealed a high-intensity signal within the right mesial thalamus (Fig. 1D). The patient was discharged without any residual deficits 5 days after the procedure.

\section{Case 2}

A 67-year-old female had a history of clipping for a ruptured left internal carotid artery-posterior communicating artery (ICA-Pcom) aneurysm and coil embolization for an unruptured right ICA-Pcom aneurysm. An unruptured basilar-tip aneurysm gradually developed with a bleb (Fig. 2A). She received aspirin $100 \mathrm{mg}$ /day and clopidogrel $75 \mathrm{mg} /$ day for 2 weeks prior to coil embolization. During the procedure, she received 8000 units of intravenous heparin to maintain an activated clotting time of between 250 and 300 seconds. She also received $80 \mathrm{mg}$ propofol and $180 \mathrm{mg}$ vecuronium during the procedure. We confirmed the presence of TPA originating from the bilateral posterior cerebral arteries (PCAs) (Fig. 2B). The patient underwent a stentassisted coil embolization and a subsequent cerebral angiography showed that the TPA was patent (Fig. 2C). She experienced memory loss for 4 days between the date of admission and the date of surgery, and could not recall the date, location, reason for admission, or the name of her neurosurgeon, whom she 


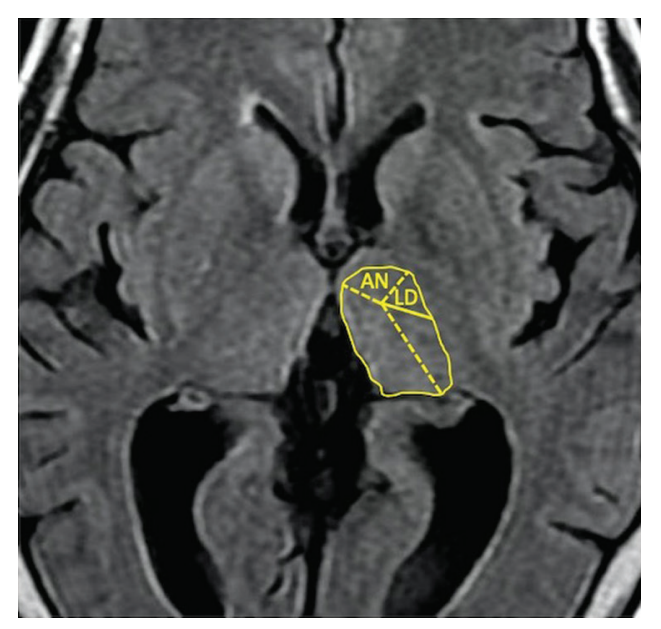

Fig. 3 The schema of AN and LD of thalamus. Dashed line showed the internal medullary lamina of thalamus. AN: anterior nucleus, LD: laterodorsal nucleus.

had met the day before coil embolization after recovering from the anesthesia. For example, she could not remember why she was in the hospital despite often being reminded by medical staff every time. She received argatroban and edaravone for the ischemic symptoms. Her anterograde amnesia disappeared 14 hours after coil embolization and a DWI revealed a high-intensity signal within the bilateral anterior nucleus of the thalamus (Fig. 2D). The patient was discharged 5 days after coil embolization without any neurological deficits.

\section{Discussion}

TGA is defined as anterograde and in some cases retrograde amnesia, with no accompanying disorder of consciousness or loss of personal identity, no other cognitive impairments, or focal neurological or epileptic signs, no recent history of head trauma, and a full resolution within 24 hours. The possibility of epilepsy, adverse effects of medication, and metabolic disorders should be eliminated during the differential diagnosis for TGA. The possibility of adverse effects of medication, such as anesthetics, was lower among the various differential diagnoses because our patients recovered fully after the procedure.

Previous reports have suggested that ischemic changes in the hippocampus or anterior nucleus of the thalamus are responsible for TGA. ${ }^{2,4)}$ Few studies have described TGA induced by coil embolization for posterior-circulation cerebral aneurysms. ${ }^{1)}$ GraffRadford et al. ${ }^{3)}$ reported TGA after coiling of a basilar artery-superior cerebellar artery aneurysm and that the symptoms fully resolved within 24 hours.
The MRI of our patients demonstrated ischemic changes in the anterior nucleus of the thalamus, which plays a role in the "Papez circuit" memory system. The thalamic vascular supply is classically categorized into four regions: anterior, paramedian, inferolateral, and posterior. ${ }^{5-7)}$ The anterior regions, including the anterior nucleus (Fig. 3), are mainly supplied by the premammillary artery, which arises from the Pcom. The paramedian regions are supplied by the TPA, which arises from the P1 segment of the PCA. Although the premammillary artery mainly feeds the anterior nucleus, the Pcom was hypoplastic in our two cases. Therefore, TPA can be a dominant feeder for the anterior nucleus, and ischemic changes of the anterior nucleus induced by micro-embolism during the coil embolization procedure could be considered as the etiology of TGA symptoms. TGA occurring after coil embolization may be underdiagnosed because of the global anesthesia effect. The status of both the Pcom and TPA should be carefully confirmed before endovascular surgery for an aneurysm of the posterior circulation, including a basilar-tip aneurysm. The symptoms of TGA occurring after coil embolization should be carefully investigated, and such patients may require anticoagulation therapy.

\section{Conclusion}

TGA aneurysm is a rare complication of coil embolization for a posterior circulation cerebral aneurysm. The anterior nucleus of the thalamus, which is supplied by the TPA and premammillary artery, plays a crucial role in ischemic symptoms such as memory disorders. The preservation of the TPA and premammillary artery should be confirmed by cerebral angiography after the coil embolization procedure.

\section{Conflicts of Interest Disclosure}

All authors have no conflict of interest.

\section{References}

1) Foss-Skiftesvik J, Snoer AH, Wagner A, Hauerberg J: Transient global amnesia after cerebral angiography still occurs: case report and literature review. Radiol Case Rep 9: 988, 2014

2) Goldenberg G, Podreka I, Pfaffelmeyer N, Wessely P, Deecke L: Thalamic ischemia in transient global amnesia: a SPECT study. Neurology 41: 1748-1752, 1991

3) Graff-Radford J, Clapp AJ, Lanzino G, Rabinstein AA: Transient amnesia after coiling of a posterior circulation aneurysm. Neurocrit Care 18: 245-247, 2013 
4) Lewis SL: Aetiology of transient global amnesia. Lancet 352: 397-399, 1998

5) Amin OS, Shwani SS, Zangana HM, Hussein EMH Ameen NA: Bilateral infarction of paramedian thalami: A report of two cases of artery of percheron occlusion and review of the literature. BMJ Case Rep 2011: bcr0920103304, 2011

6) Cassourret G, Prunet B, Sbardella F, Bordes J, Maurin O, Boret $\mathrm{H}$ : Ischemic stroke of the artery of percheron with normal initial mri: a case report. Case Rep Med 2010: 425734, 2010
7) Lazzaro NA, Wright B, Castillo M, et al.: Artery of percheron infarction: Imaging patterns and clinical spectrum. AJNR Am J Neuroradiol 31: 1283-1289, 2010

Corresponding author: Yoshinobu Kamio, MD, PhD Department of Neurosurgery, Hamamatsu University School of Medicine, 1-20-1 Handayama, Higashi-ku, Hamamatsu, Shizuoka 4313192 Japan. e-mail: ykamio@hama-med.ac.jp 